\title{
A CASE REPORT: THE ROLE OF PROSTATE-SPECIFIC MEMBRANE ANTIGEN LABELED THERANOSTIC AGENTS IN THE DIAGNOSIS AND TREATMENT OF PROSTATE CANCER
}

\author{
Ali Rıza Avul' ${ }^{1}$ Büşra Özdemir² ${ }^{2}$ Gülay Durmuş Altun² \\ ${ }^{1}$ Trakya University School of Medicine, Edirne, TURKEY \\ ${ }^{2}$ Department of Nuclear Medicine, Trakya University Faculty of Medicine, Edirne, TURKEY
}

\section{ABSTRACT}

Aims: Prostate cancer is one of the most prevalent cancers worldwide and sometimes it can be deadly which brings out a high quantity of the prostate specific membrane antigen. Gallium-68 prostate specific membrane antigen PET scan is used to detect primary tumors and metastases of prostate cancer. In addition, Lutetium-177 prostate-specific membrane antigen is used for treatment in some special cases. Our case report aims to show the roles of prostate specific membrane antigen labeled theranostic agents in the diagnosis, staging, and treatment of prostate cancer and evaluate the response to the treatment of metastatic castration-resistant prostate cancer.

Case Report: A 78-year-old male patient was admitted to Trakya University Hospital Nuclear Medicine Department with the diagnosis of prostate cancer for cancer staging and treatment. Gallium-68 prostate specific membrane antigen was firstly used to detect any metastases and then to evaluate response to the treatment. Lutetium-177 prostate specific membrane antigen was used for treatment.

Conclusion: After 3 cures of Lutetium-177 therapy, the patient underwent Gallium - 68 prostate specific membrane antigen PET which demonstrated regression of the metastatic tumor. There was a decrease in the uptake of Gallium-68 prostate specific membrane antigen in the primary tumor and lymph nodes metastases. Also, bone metastases have been cleared. Hence, Lutetium-177 seems to be a promising treatment modality to treat metastatic prostate cancer.

Keywords: Prostate cancer, theranostic, lutetium

\section{INTRODUCTION}

Cases of prostate cancer are frequently reported and metastatic prostate cancer has a high mortality rate. According to the American Cancer Society's statistics the 5-year survival of metastatic prostate cancer is $30 \%(1,2)$.

Prostate cancer can metastasize to lymph nodes, bones, rarely to lung, skin and liver. It is very important to detect primary tumor before the metastasis. According to studies, the survival rate of prostate cancer with minimal metastases was less than 48 months and the survival rate of metastatic prostate cancer that is resistant to androgen suppression therapy was less than 24 months (3).

Prostate specific membrane antigen (PSMA) is a kind of cell surface receptor which is expressed in various tissues like salivary glands, kidneys, liver, prosta- te, small intestine and incomparably highly expressed in prostate cancer cells, particularly in its metastatic form. PSMA is non-secretory and membrane-bound. Binding of ligands to PSMA leads to internalization via clathrin-coated pits and these properties make PSMA an ideal target for drug delivery for both diagnostic and therapeutic purposes (4).

PSMA-labeled Gallium(Ga)-68 PET scan is used to detect primary tumors and its metastases with high accuracy for staging. According to studies, Gallium-68 PSMA PET scan can detect very small metastases which are not detected by other scanning techniques like computed tomography (CT) and magnetic resonance imaging (MRI) (5). In addition, it is very successful in showing recurrence in early periods. Afshar et al. (6) found that the positive predictive value of $\mathrm{Ga}-68$ PET scan is $100 \%$ and sensitivity is $88.1 \%$ for detecting recurrence of prostate cancer. Gallium-68 PSMA PET scan can also help us to evaluate the response of cancer to treatments. 
Newly developed ligands can bind to the extracellular part of PSMA which can help us to scan living prostatic cells. PSMA scan is indicated in patients with a Gleason score of 7 or more, before radiotherapy or in patients that are considered biochemically recurrent cases. PSMA scan should be conducted 2 weeks after chemotherapy and hormone therapy or 6 weeks after radiotherapy (7).

In addition to diagnostic purpose, Lutetium (Lu)177 PSMA can act as a therapeutic agent in metastatic castration-resistant prostate cancer (mCRPC). According to studies Lu-177 PSMA is very effective and safe in treatment. Emmett et al. (8) found that 28 months following Lu-177 PSMA therapy, the survival rate was $78.6 \%$.

Therefore, PSMA can potentially act as a diagnostic and therapeutic agent. If PMSA is labeled with Ga-68, it is used for diagnosis, whereas if it is labeled with Lu177, which has more energy than Ga-68, it is used for treatment. Depending on the type of peptide attached to PSMA receptors, PSMA can be a theranostic agent in staging and treating patients with prostate cancer at the same time.

\section{CASE REPORT}

A 78-year-old male patient presented to Trakya University Nuclear Medicine Department with the diagnosis of prostate cancer and was referred to scan distant metastasis in February 2017. The patient had prostate adenocarcinoma and the Gleason score was found as $4+4$ in January 2017.

After the diagnosis, the patient was scanned for bone metastasis in a different clinic by Technetium 99m MDP SPECT and metastases at the spine, right sacroiliac joint, head of the right femur and right side of rib were detected. Following metastasis scan, both of the patient's testicles were removed at a different medical center to reduce testosterone levels. The patient was followed-up by imaging PET and blood PSA levels.

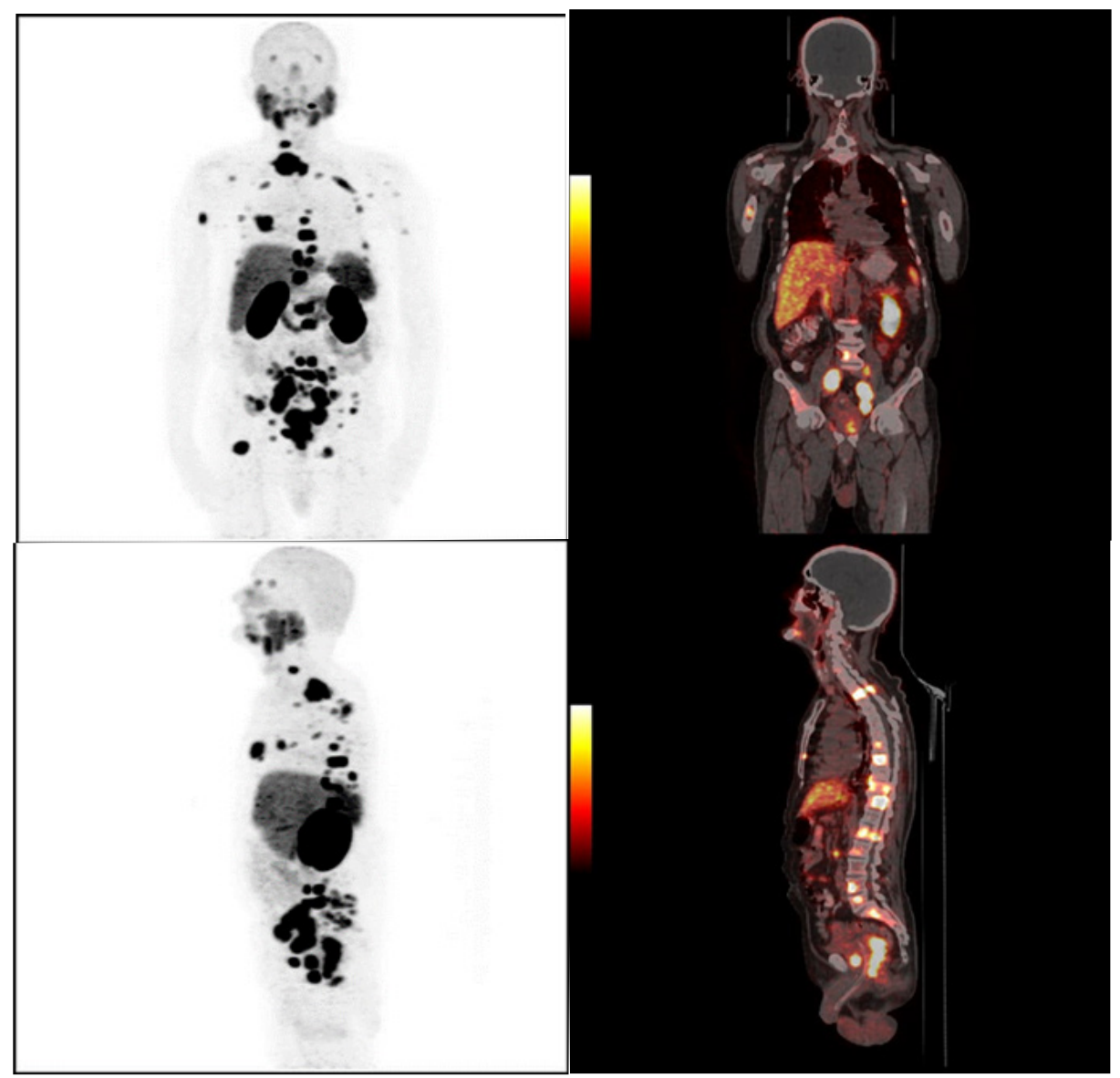

Figure 1: Ga-68 PSMA PET scan in February 2017. 


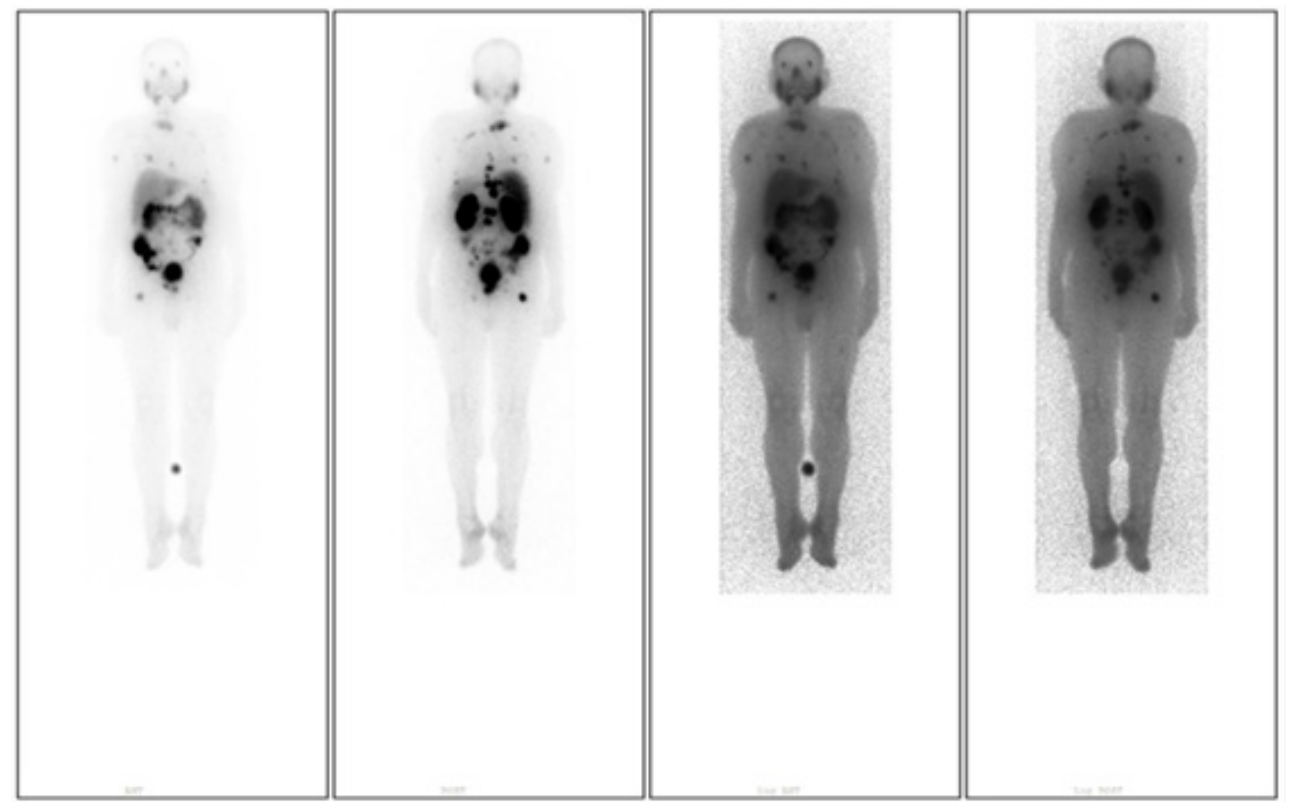

Figure 2: Day 1, whole body scan following Lu 177 therapy.

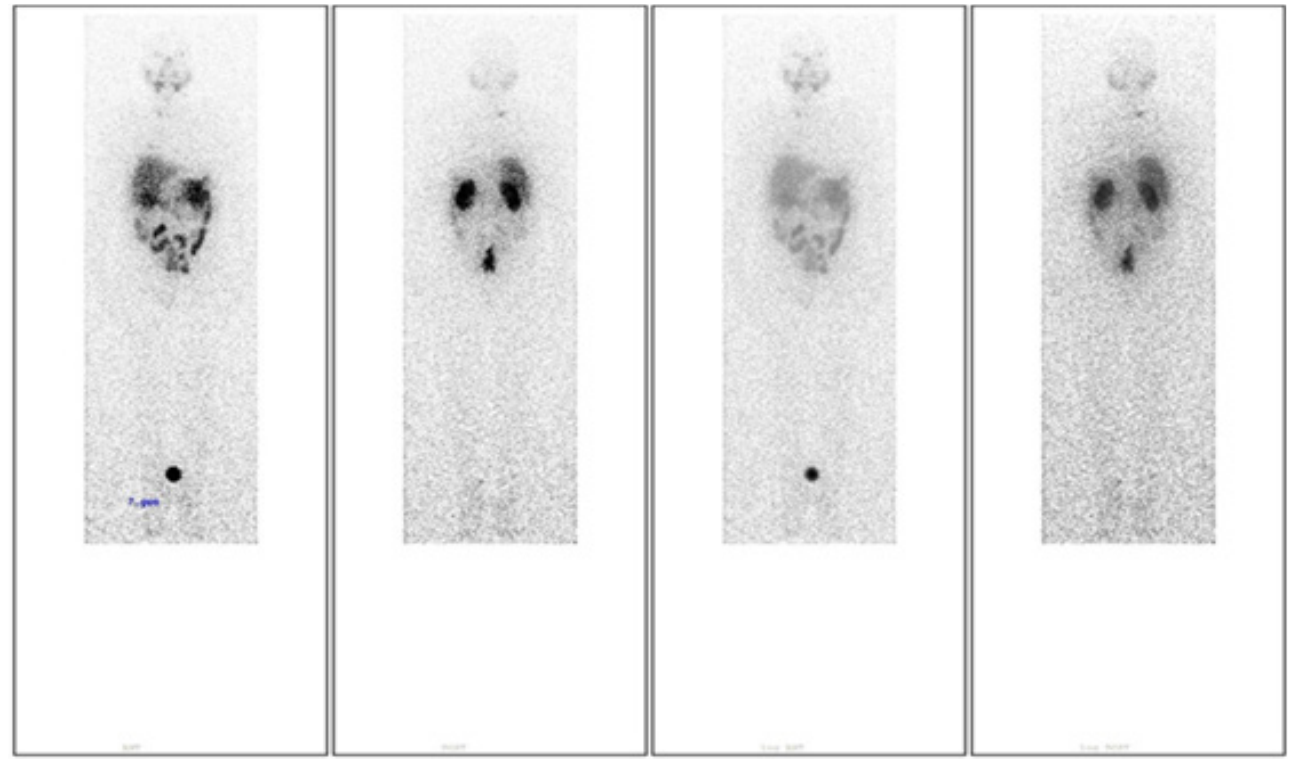

Figure 3: Whole body scan following $3 \mathrm{Lu}$-177 therapies.

In February 2017, the patient underwent Ga-68 PSMA PET in Trakya University Hospital. The primary prostate tumor metastasized to various lymph nodes, spines, hipbone, sacroiliac joint, right femur, right side of ribs, both scapulas and right humerus (Figure 1). The patient was diagnosed with mCRPC and clinicians decided to start Lu-177 PSMA therapy. The whole body scan on the first day of Lu-177 PSMA therapy and after the treatment regime are demonstrated in Figure 3 and Figure 4 respectively.
After 3 cycles of Lu-PSMA treatment, the patient was assessed for the therapeutic efficacy of Lu-PSMA treatment by Ga-68 PSMA PET (Figure 4). The uptake of Ga-68 PSMA reduced the size of the tumor. There were a lot of pelvic lymph node metastases and all of them were smaller than $1 \mathrm{~cm}$. No bone metastasis was detected. 


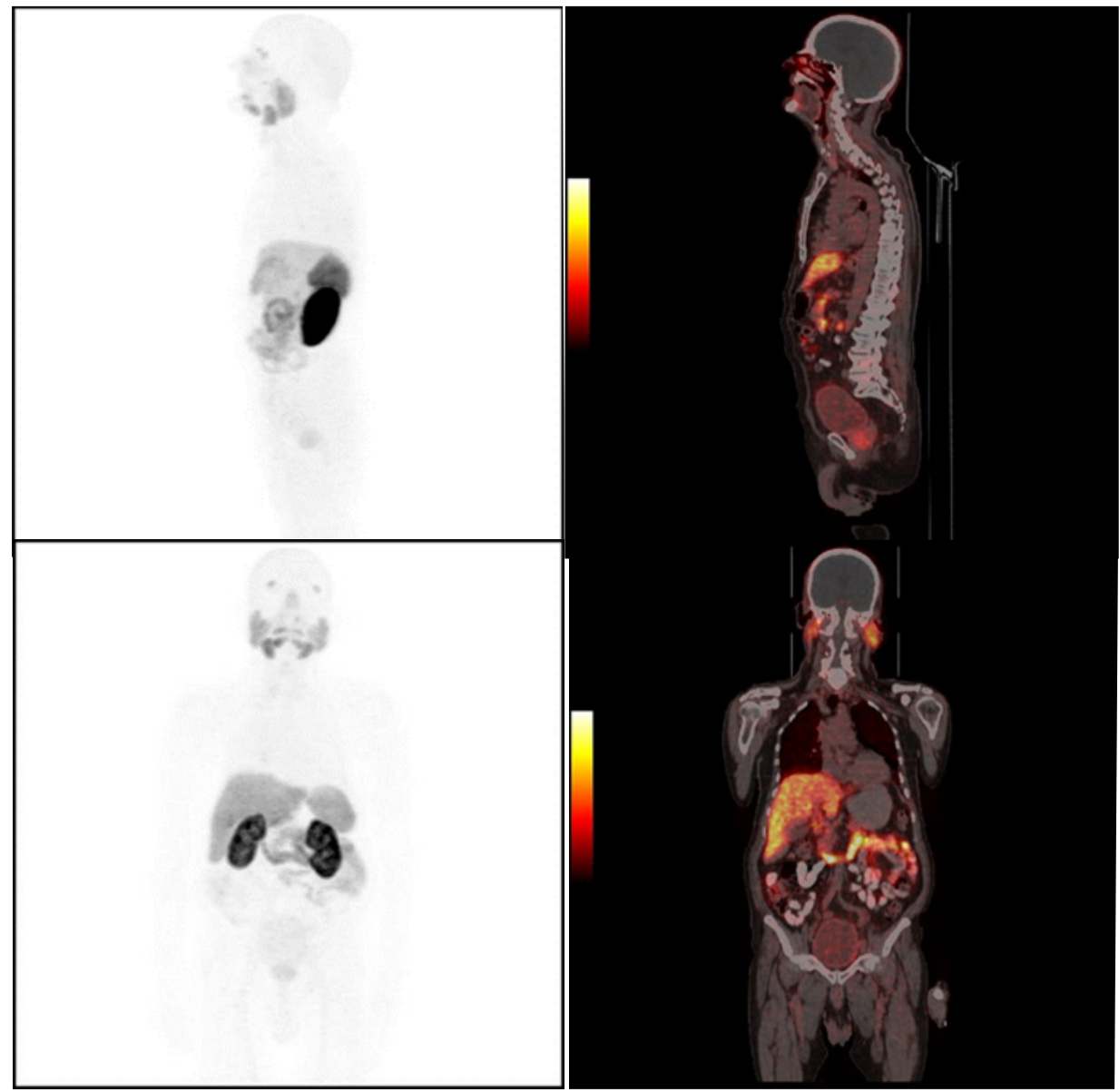

Figure 4: Ga-68 PSMA PET scan following 3 cycles of Lu-PSMA treatment to assess treatment efficacy.

Ga-68 PSMA PET scan was found very effective in detecting metastasis (9). In the present case study, it detected a $6 \mathrm{~mm}$ large lymph node metastasis, which could not be detected by CT or MRI.

PSA was used as a marker of treatment response (10). Blood total PSA level was $83.59 \mathrm{ng} / \mathrm{ml}$ in March 2017 and the total PSA was $156 \mathrm{ng} / \mathrm{ml}$ in May 2017. After 3 cycles of Lu-PSMA therapies, patient's blood total PSA level was $0.7 \mathrm{ng} / \mathrm{ml}$ and free PSA level was 0.0037 $\mathrm{ng} / \mathrm{ml}$ in December 2017.

Before the therapy, there was very active primary tumor in prostate $(\mathrm{SUVmax}=16.2)$ and the tumor metastasized to a number of lymph nodes and pelvis. Some of the lymph node metastases were multiple in colonies and bigger than $1 \mathrm{~cm}$ such as in presacral, right pararectal and internal iliac lymph nodes. Moreover, some of the lymph node metastases were bigger than $4 \mathrm{~cm}$ like in internal, external and common iliac lymph nodes.
Additionally, there were a lot of bone metastases in $\mathrm{C} 1$ and $\mathrm{C} 2$.

After this therapy, primary tumor activity regressed (SUV max = 5.5). The bone metastasis could not be detected on the PET scan. In addition, the lymph node involvement was regressed. All of the lymph node metastases observed were smaller than $1 \mathrm{~cm}$. 


\section{DISCUSSION}

Prostate cancer is the most common type of cancer in men (1). It can metastasize to various tissues. Prostate cancer should be detected before it metastasises, otherwise it can be lethal (2). It is very important to detect the primary tumor and their metastases and treat properly if there are metastases. Androgen deprivation therapy is applied to reduce testosterone levels since testosterone is the cause of prostate cancer cell growth and proliferation. But in some cases the tumor cells are resistant to androgen deprivation therapy, thus are called mCRPC (1). The survival of these cases is less than 24 months (3). Also it is very important to monitor the treatment properly. Prostate cancer may reoccur and this recurrence may not be detected because there may be some recurrences which are too small to detect by any radiological imaging or doctors may not be suspected of recurrence because the PSA level is low.

The treatment and monitoring methods may be inadequate in some cases. Therefore, new methods are required to treat patients who do not respond to conventional therapy. If only PSA based screening or imaging is used, some recurrent cases may be missed. According to various studies, theranostic agents are more sensitive for screening and more effective for treatment in $\mathrm{mCRPC}$ cases (5-8).

In this case, it is observed that theranostic agents can reduce the activity of prostate cancer in mCRPC and it is found that Ga-68 PSMA is reliable to detect metastases and monitoring treatment. In our case a 6 mm large lesion was observed with Ga-68 PSMA PET, which could not be observed in CT. Ga-68 PSMA PET can show recurrences, although PSA levels are low $(0.2$ $\mathrm{ng} / \mathrm{ml}$ to $10 \mathrm{ng} / \mathrm{ml}$ ) (11). But the data and experience is insufficient to state Lu-177 PSMA has a positive effect on survival of mCRPC (12).

In this case, results showed that Lu-177 PSMA therapy is effective in the treatment of metastatic prostate cancer. The patient demonstrated regression of the primary and metastatic tumor. Theranostic agents were also effective in cancer diagnosis and monitoring treatment efficacy. Our results herald prospective studies with larger sample size and longer follow-up period to further discern the treatment efficacy and detection rate of PSMA in prostate cancer.

As a conclusion it should be noted that $\mathrm{Lu}-177$ PSMA is showing exciting treatment responses in men with $\mathrm{mCRPC}$ and has an important future role in the treatment of prostate cancer (12).

\section{Ethics Committee Approval: N/A}

Informed Consent: Written informed consent was obtained from the participant of this study.

Conflict of Interest: The authors declared no conflict of interest.

Author contributions: Concept: ARA, BÖ, GDA. Design: ŞYS. Supervision: ARA, BÖ, GDA. Resources: ARA, BÖ, GDA. Materials: ARA, BÖ, GDA. Data collection and/or processing: ARA, BÖ, GDA. Analysis and/or Interpretation: ARA, BÖ, GDA. Literature Search: ARA, BÖ, GDA. Writing Manuscript: ARA, BÖ, GDA. Critical Review: ARA, BÖ, GDA.

Financial disclosure: The authors declared that this study received no financial support.

\section{REFERENCES}

1. Mottet N, Bellmunt J, Briers E et al. Guidelines on prostate cancer, 2015. Available from: URL: https:// uroweb.org/wp-content/uploads/09-Prostate-Cancer_ LR.pdf.

2. American Cancer Society. Cancer facts \& figures 2018. Available from: URL: https://www.cancer. org/content/dam/cancer-org/research/cancer-facts-and-statistics/annual-cancer-facts-and-figures/2018/cancer-facts-and-figures-2018.pdf.

3. Crawford ED, Petrylak D, Sartor O. Navigating the evolving therapeutic landscape in advanced prostate cancer. Urol Oncol 2017;35:1-13.

4. Teo MY, Morris MJ. Prostate-specific membrane antigen-directed therapy for metastatic castration-resistant prostate cancer. Cancer J 2016;22(5):347-52.

5. Giesel FL, Fiedler H, Stefanova $M$ et al. PSMA PET/ CT with Glu-urea-Lys-(Ahx)- $\left[{ }^{68} \mathrm{Ga}\right.$ (HBED-CC)] versus $3 \mathrm{D}$ CT volumetric lymph node assessment in recurrent prostate cancer. Eur J Nucl Med Mol Imaging 2015;42(12):1794-800.

6. Afshar-Oromieh A, Avtzi E, Giesel FL et al. The diagnostic value ofPET/CT imaging with the (68) Ga-labelled PSMA ligand HBED-CC in the diagnosis of recurrent prostate cancer. Eur J Nucl Med Mol Imaging 2015;42(2):197-209. 
7. Durmuş Altun-Üstün (2017) Nükleer Tıp Görüntüleme Teknikleri Ders Kitabı: Nobel Tip Kitapevleri, 2017

8. Emmett L, Willowson K, Violet J et al. Lutetium 177 PSMA radionuclide therapy for men with prostate cancer: a review of the current literature and discussion of practical aspects of therapy. J Med Radiat Sci 2017;64(1):52-60.

9. Udovicich C, Perera M, Hofman M et al. 68Ga-prostate-specific membrane antigen-positron emission tomography/computed tomography in advanced prostate cancer: current state and future trends. Prostate International 2017;5(4):125-9.

10. Ahmadzadehfar H, Wegen S, Yordanova A et al. Overall survival and response pattern of castration-resistant metastatic prostate cancer to multiple cycles of radioligand therapy using [177Lu]Lu-PSMA. Eur J Nucl Med Mol Imaging 2017;44(9):1448-54.

11. Rauscher I, Maurer T, Fendler WP et al. 68Ga-PSMA ligand PET/CT in patients with prostate cancer: how we review and report. Cancer Imaging 2016;16:14.

12. Kabasakal L, Toklu T, Yeyin N. Lu-177-PSMA-617 prostate-specific membrane antigen inhibitor therapy in patients with castration-resistant prostate cancer: stability, bio-distribution and dosimetry. Mol Imaging Radionucl Ther 2017;26(2):62-8. 